\title{
Small surfaces of Willmore type in Riemannian manifolds
}

\author{
Tobias Lamm* \\ Department of Mathematics, University of British Columbia, 1984 \\ Mathematics Road, Vancouver, BC V6T 1Z2, Canada \\ Jan Metzger \\ Albert-Einstein-Institut, Am Mühlenberg 1, 14476 Potsdam, Germany \\ Universität Freiburg, Institut für Reine Mathematik, \\ Eckerstr. 1, 79104 Freiburg, Germany
}

\begin{abstract}
In this paper we investigate the properties of small surfaces of Willmore type in Riemannian manifolds. By small surfaces we mean topological spheres contained in a geodesic ball of small enough radius. In particular, we show that if there exist such surfaces with positive mean curvature in the geodesic ball $B_{r}(p)$ for arbitrarily small radius $r$ around a point $p$ in the Riemannian manifold, then the scalar curvature must have a critical point at $p$.

As a byproduct of our estimates we obtain a strengthened version of the non-existence result of Mondino [8] that implies the non-existence of certain critical points of the Willmore functional in regions where the scalar curvature is non-zero.
\end{abstract}

\section{Introduction}

In a previous paper [6] Willmore type surfaces were introduced and foliations of asymptotically flat manifolds by such surfaces were studied. In this paper we turn to the local situation and consider Willmore type surfaces in small geodesic balls in Riemannian manifolds. The focus is on a priori estimates for such surfaces under the assumption of positive mean curvature and a

\footnotetext{
*Partially supported by a PIMS Postdoctoral Fellowship.
} 
growth condition for the Lagrange parameter. As an application of these estimates we derive a necessary condition for the existence of such surfaces.

By surfaces of Willmore type we mean surfaces $\Sigma$ that are critical for the Willmore functional

$$
\mathcal{W}(\Sigma)=\frac{1}{2} \int_{\Sigma} H^{2} \mathrm{~d} \mu
$$

subject to an area constraint $|\Sigma|=a$, where $a$ is some fixed constant. These surfaces are solutions of the Euler-Lagrange equation

$$
\Delta H+H|\stackrel{\AA}{A}|^{2}+H \operatorname{Ric}(\nu, \nu)+\lambda H=0
$$

where $\lambda \in \mathbf{R}$ is the Lagrange parameter, $H$ the mean curvature of $\Sigma, \AA$ denotes the traceless part of the second fundamental form, Ric refers to the Ricci curvature of the ambient manifold, $\nu$ is the normal of $\Sigma$ and $\Delta$ denotes the Laplace-Beltrami operator on $\Sigma$. In particular these surfaces are a generalization of Willmore surfaces that are critical for $\mathcal{W}$ without constraint and therefore satisfy the equation

$$
\Delta H+H|\AA|^{2}+H \operatorname{Ric}(\nu, \nu)=0 .
$$

We note here that there are other functionals that can be considered as generalizations of the Willmore functional in Riemannian manifolds, for example the functional $\mathcal{U}$ introduced in section 2 could be used (see [12]).

The precise statement of the main result of this paper is the following:

Theorem 1.1. Assume that $(M, g)$ is a Riemannian manifold such that the curvature and the first two derivatives of the curvature are bounded. Then there exist $\varepsilon_{0}>0$ and $C<\infty$, depending only on these bounds, with the following properties.

Given $p$ in $M$ and assume that there is $r_{0}>0$ such that for each $r \in\left(0, r_{0}\right]$ there exists a surface $\Sigma_{r}$ of Willmore type in $B_{r}(p)$, that is on $\Sigma_{r}$ we have

$$
\Delta H+H|\stackrel{\leftrightarrow}{A}|^{2}+H \operatorname{Ric}(\nu, \nu)+H \lambda_{r}=0,
$$

such that in addition the following conditions are satisfied for $\varepsilon<\varepsilon_{0}$ :

1. $\Sigma_{r}$ is a topological sphere,

2. $\lambda_{r} \geq-\varepsilon /\left|\Sigma_{r}\right|$, and

3. $H>0$ on $\Sigma_{r}$. 
Then

$$
\lim _{r \rightarrow 0}\left|\lambda_{r}+\frac{1}{3} \operatorname{Sc}(p)\right|=0
$$

Here $\operatorname{Sc}(p)$ denotes the Scalar curvature of $M$ at the point $p$. Furthermore

$$
\nabla \mathrm{Sc}(p)=0
$$

where $\nabla \mathrm{Sc}(p)$ denotes the gradient of the scalar curvature of $M$ at $p$.

The first claim is proved in section 3 as a consequence of the a priori estimates for surfaces of Willmore type derived there. Section 4 is devoted to the proof of the second claim.

For surfaces of constant mean curvature (CMC), that is surfaces satisfying $H=$ const, analogous properties have been derived. Ye showed that if there locally exists a regular foliation by CMC surfaces near a point $p$, then $p$ is necessarily a critical point of the scalar curvature. For the detailed statement including the technical conditions we refer to [13, Theorem 2.1] (see also [14]). There are further results in this direction by Druet [3] and Nardulli [9] where the expansion of the isoperimetric profile of a Riemannian manifold is computed. This computation shows that isoperimetric surfaces also concentrate near critical points of the scalar curvature.

Indeed, it has been shown by Ye in [13] that near non-degenerate critical points of the scalar curvature there exist spherical surfaces with arbitrarily large mean curvature, or equivalently, arbitrarily small area. We expect that a similar statement is true for surfaces of Willmore type, namely that near a non-degenerate critical point of the scalar curvature there exist surfaces of Willmore type with arbitrarily small area. We will address this elsewhere.

An immediate corollary of theorem 1.1, is the following strengthened version of the non-existence result of Mondino [8, Theorem 1.3] for Willmore surfaces. These surfaces are of Willmore type with multiplier $\lambda=0$, and thus the previous theorem is applicable.

Corollary 1.2. Let $(M, g)$ be a Riemannian manifold as in theorem 1.1 and let $p \in M$. If $\operatorname{Sc}(p) \neq 0$ or $\nabla \mathrm{Sc}(p) \neq 0$ then there exists $r>0$ such that $B_{r}(p)$ does not contain spherical Willmore surfaces with positive mean curvature.

We conclude the paper with section 5, where the a priori estimates are used to calculate the expansion of the Willmore functional on surfaces as in theorem 1.1. More precisely, we show that for these surfaces we have

$$
\mathcal{W}(\Sigma)=8 \pi-\frac{|\Sigma|}{3} \operatorname{Sc}(p)+O(r|\Sigma|) .
$$


This is analogous to the expansion derived by Mondino [8, Proposition 3.1] for perturbed spheres.

\section{Preliminaries}

In this section we describe our notation and we provide some tools in order to analyze small surfaces in a Riemannian manifold.

\subsection{Notation}

We consider surfaces $\Sigma$ in a three dimensional Riemannian manifold $(M, g)$, where $g$ denotes the metric on $M$. We denote by $\nabla$ the induced Levi-CivitaConnection, by Ric its Ricci-curvature and by Sc its scalar curvature.

If $p \in M$ and $\rho<\operatorname{inj}(M, g, p)$, the injectivity radius of $(M, g)$ at $p$, we can introduce Riemannian normal coordinates on $B_{\rho}(p)$, the geodesic ball of radius $\rho$ around $p$. These are given by the map

$$
\Phi: B_{\rho}^{E}(0) \rightarrow B_{\rho}(p): x \mapsto \exp _{p}(x)
$$

where $B_{\rho}^{E}(0)$ is the Euclidean ball of radius $\rho$ in $\mathbf{R}^{3} \cong T_{p} M$. In these coordinates the metric $g$ satisfies

$$
g=g^{E}+h
$$

where $g^{E}$ denotes the Euclidean metric and $h$ satisfies

$$
|x|^{-2}|h|+|x|^{-1}|\partial h|+\left|\partial^{2} h\right| \leq h_{0}
$$

for all $x \in B_{\rho}^{E}(0)$. Here $h_{0}$ is a constant depending only on the maximum of $\mid$ Ric $|,| \nabla$ Ric $\mid$ and $\mid \nabla^{2}$ Ric $\mid$ in $B_{\rho}(p)$. More detailed expansions are not needed here but can be found in [10, Lemma V.3.4]. For our purposes it is sufficient to consider $M=B_{\rho}^{E}(0)$ to be equipped with the two metrics $g$ and $g^{E}$. We will denote $B_{\rho}=B_{\rho}^{E}(0)$ in the sequel.

If $\Sigma \subset B_{\rho}(p)$ is a surface, we denote its normal vector by $\nu$, its induced metric by $\gamma$ and its second fundamental form by $A$. The mean curvature of $\Sigma$ is denoted by $H=\operatorname{tr}_{\gamma} A$ and the traceless part of the second fundamental form by $\AA=A-\frac{1}{2} H \gamma$. Furthermore, $\mathrm{d} \mu$ denotes the measure on $\Sigma$. Note that also the Euclidean metric induces a full set of geometric quantities on $\Sigma$, which will be distinguished by the superscript ${ }^{E}$, for example $\nu^{E}, A^{E}$, $H^{E}$, etc. All geometric quantities which we leave undecorated correspond to the metric $g$. 


\subsection{The Willmore Functional}

Assume that $\Sigma \subset M$. Then we consider the Willmore functional on $\Sigma$, that is the functional

$$
\mathcal{W}(\Sigma)=\frac{1}{2} \int_{\Sigma} H^{2} \mathrm{~d} \mu
$$

We say that a surface is of Willmore type with multiplier $\lambda \in \mathbf{R}$ if it satisfies the equation

$$
\Delta H+H|\stackrel{\AA}{A}|^{2}+H \operatorname{Ric}(\nu, \nu)+\lambda H=0 .
$$

Here $\Delta$ denotes the Laplace-Beltrami operator on $\Sigma$ and Ric refers to the Ricci-curvature of the ambient metric $g$ as before. Equation (2.3) arises as the Euler-Lagrange equation for the the following variational problem:

$$
\begin{cases}\text { Minimize } & \mathcal{W}(\Sigma) \\ \text { subject to } & |\Sigma|=a\end{cases}
$$

where $a$ is a given constant. The parameter $\lambda$ in (2.3) is then just the Lagrange-parameter of the critical point. For a derivation of this and further motivation we refer to [6].

Denoting by ${ }^{{ }^{5}}$ Sc the scalar curvature of $\Sigma$, the Gauss equation implies that

$$
{ }^{\Sigma} \mathrm{Sc}=\operatorname{Sc}-2 \operatorname{Ric}(\nu, \nu)+\frac{1}{2} H^{2}-|\stackrel{\circ}{A}|^{2} .
$$

Integrating this equation on $\Sigma$ yields the identity

$$
\mathcal{W}(\Sigma)=8 \pi(1-q(\Sigma))+\mathcal{U}(\Sigma)+\mathcal{V}(\Sigma)
$$

where $q(\Sigma)$ denotes the genus of $\Sigma$,

$$
\begin{aligned}
& \mathcal{U}(\Sigma)=\int_{\Sigma}|\AA|^{2} \mathrm{~d} \mu, \quad \text { and } \\
& \mathcal{V}(\Sigma)=2 \int_{\Sigma} G(\nu, \nu) \mathrm{d} \mu .
\end{aligned}
$$

Here $G=\operatorname{Ric}-\frac{1}{2}$ Sc $g$ denotes the Einstein tensor of $M$. This splitting was used in [6] to obtain a priori estimates for the position of Willmore type surfaces in asymptotically flat manifolds and shall also play an important role in section 4 . 


\subsection{Small surfaces}

Since we compare the geometry of a surface $\Sigma$ with respect to the ambient metrics $g$ and $g^{E}$ we need the following lemma.

Lemma 2.1. Let $g=g^{E}+h$ on $B_{\rho}$ be given. Then there exists a constant $C$ depending only on $\rho$ and $h_{0}$ from equation (2.2) such that for all surfaces $\Sigma \subset B_{r}$ with $r<\rho$ we have

$$
\begin{aligned}
& \left|\gamma-\gamma^{E}\right| \leq C|x|^{2}, \\
& \left|\mathrm{~d} \mu-\mathrm{d} \mu^{E}\right| \leq C|x|^{2}, \\
& \left|\nu-\nu^{E}\right| \leq C|x|^{2}, \quad \text { and } \\
& \left|A-A^{E}\right| \leq C\left(|x|+|x|^{2}|A|\right) .
\end{aligned}
$$

In the sequel we will use the big- $O$ notation. By the statement $f=O\left(r^{\alpha}\right)$ we mean that for any $r_{0}>0$ there exists a constant $C<\infty$ such that $|f| \leq C r^{\alpha}$ provided that $r<r_{0}$.

Observe that the area of a surface in $B_{\rho}$ is bounded in terms of $\rho$ and the Willmore functional. This lemma is a slight generalization of [11, Lemma 1.1].

Lemma 2.2. Let $g=g^{E}+h$ on $B_{\rho}$ be given. Then there exists $0<\rho_{0}<\rho$ and a constant $C$ depending only on $\rho$ and $h_{0}$ such that for all surfaces $\Sigma \subset B_{r}$ with $r<\rho_{0}$ we have

$$
|\Sigma| \leq C r^{2} \int_{\Sigma} H^{2} \mathrm{~d} \mu .
$$

Proof. Let $\Sigma \subset B_{r}$ be a hypersurface for some $r \leq \rho$. We consider the position vector field $x$ on $B_{\rho}$. Then we have

$$
\operatorname{div}_{\Sigma} x=2+O(|x|)
$$

where $\operatorname{div}_{\Sigma}$ means the tangential divergence along $\Sigma$. Integrating this relation yields

$$
2|\Sigma|=\int_{\Sigma} \operatorname{div}_{\Sigma} x \mathrm{~d} \mu+|\Sigma| O(r) .
$$

Since

$$
\int_{\Sigma} \operatorname{div}_{\Sigma} x \mathrm{~d} \mu=\int_{\Sigma} H\langle x, \nu\rangle \mathrm{d} \mu \leq\left(\int_{\Sigma} H^{2} \mathrm{~d} \mu\right)^{1 / 2}\left(\int_{\Sigma}|\langle x, \nu\rangle|^{2} \mathrm{~d} \mu\right)^{1 / 2}
$$


and $|\langle x, \nu\rangle| \leq r$, we find that

$$
|\Sigma| \leq r|\Sigma|^{1 / 2}\left(\int_{\Sigma} H^{2} \mathrm{~d} \mu\right)^{1 / 2}+C r|\Sigma| .
$$

Now we can fix $\rho_{0}$ small, so that for all $0<r<\rho_{0}$ the the second term on the right can be absorbed to the left. This yields the claimed inequality.

For the subsequent curvature estimates we also need a version of the MichaelSimon-Sobolev inequality. It follows from the Euclidean version of the inequality [7] in conjunction with lemma 2.1 to change to the respective quantities to the $g$-metric.

Lemma 2.3. Let $g=g^{E}+h$ on $B_{\rho}$ be given. Then there exists $0<\rho_{0}<\rho$ and a constant $C$ depending only on $\rho$ and $h_{0}$ such that for all surfaces $\Sigma \subset B_{\rho_{0}}$ with $\|H\|_{L^{2}(\Sigma)}<\infty$ and all $f \in C^{\infty}(\Sigma)$ we have

$$
\left(\int_{\Sigma} f^{2} \mathrm{~d} \mu\right)^{1 / 2} \leq C \int_{\Sigma}|\nabla f|+|H f| \mathrm{d} \mu .
$$

\subsection{Almost umbilical surfaces}

Subsequently it is necessary to approximate a given surface $\Sigma$ by a Euclidean sphere. The main tool will be the following theorem from [1] and [2]. We denote the $L^{2}$-norm of the trace free part of the second fundamental form by

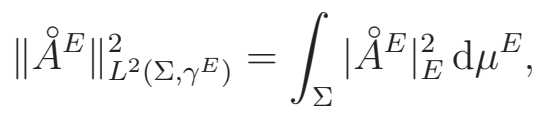

where all geometric quantities are with respect to the Euclidean background. In addition we denote by

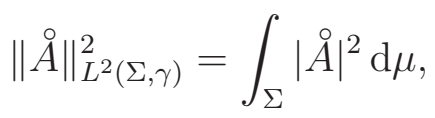

the norm of the same tensor, where all geometric quantities are calculated with respect to the background metric $g$. The following theorem is a purely Euclidean theorem.

Theorem 2.4. There exists a universal constant $C$ with the following properties. Assume that $\Sigma \subset \mathbf{R}^{3}$ is a surface with $\left\|\AA^{E}\right\|_{L^{2}\left(\Sigma, \gamma^{E}\right)}^{2}<8 \pi$. Let $R^{E}:=\sqrt{|\Sigma|^{E} / 4 \pi}$ be the Euclidean area radius of $\Sigma$ and $a^{E}:=|\Sigma|_{E}^{-1} \int_{\Sigma} x \mathrm{~d} \mu^{E}$ be the Euclidean center of gravity. Then there exists a conformal map 
$\psi: S:=S_{R^{E}}\left(a^{E}\right) \rightarrow \Sigma \subset \mathbf{R}^{3}$ with the following properties. Let $\gamma^{S}$ be the standard metric on $S, N$ the Euclidean normal vector field and $h$ the conformal factor, that is $\psi^{*} \gamma^{E}=h^{2} \gamma^{S}$. Then the following estimates hold

$$
\begin{aligned}
\left\|H^{E}-2 / R^{E}\right\|_{L^{2}\left(\Sigma, \gamma^{E}\right)} & \leq C\left\|\AA^{E}\right\|_{L^{2}\left(\Sigma, \gamma^{E}\right)} \\
\left\|\psi-\mathrm{id}_{S}\right\|_{L^{\infty}(S)} & \leq C R^{E}\left\|\AA^{E}\right\|_{L^{2}\left(\Sigma, \gamma^{E}\right)} \\
\left\|h^{2}-1\right\|_{L^{\infty}(S)} & \leq C R^{E}\left\|\AA^{E}\right\|_{L^{2}\left(\Sigma, \gamma^{E}\right)} \\
\left\|N-\nu^{E} \circ \psi\right\|_{L^{2}\left(\Sigma, \gamma^{E}\right)} & \leq C R^{E}\left\|\AA^{E}\right\|_{L^{2}\left(\Sigma, \gamma^{E}\right)} .
\end{aligned}
$$

To apply the previous theorem we need to estimate $\left\|\AA^{E}\right\|_{L^{2}\left(\Sigma, \gamma^{E}\right)}$ in terms of $\|\AA\|_{L^{2}(\Sigma, \gamma)}$. This is the content of the following lemma.

Lemma 2.5. Let $g=g^{E}+h$ on $B_{\rho}$ be given. Then there exists $0<\rho_{0}<\rho$ and a constant $C$ depending only on $\rho$ and $h_{0}$ such that for all surfaces $\Sigma \subset B_{r}$ with $r<\rho_{0}$ we have

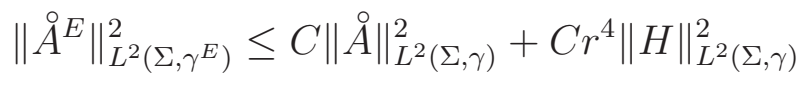

Proof. This is a straightforward consequence of lemma 2.1 and the CauchySchwarz inequality.

\section{A priori estimates}

A crucial ingredient in the proof of theorem 1.1 is an estimate for the $L^{2}$ norm of the traceless part of the second fundamental form of $\Sigma$. This allows us to control the shape of the surface $\Sigma$ in view of theorem 2.4.

Throughout this section we assume that the metric $g=g^{E}+h$ is fixed on $B_{\rho}$. We furthermore assume that $\rho$ is chosen so small that lemmas $2.1,2.2$ and 2.3 can be applied to any surface in $B_{\rho}$. We allow $\rho$ to shrink as it becomes necessary. All surfaces we consider here are of Willmore type, i.e. they satisfy equation (1.1) for some $\lambda$ and are furthermore contained in $B_{\rho}$. Subsequently all constants $C$ may depend on $\rho$ and $h_{0}$ without further notice. In addition these constants are allowed to change from line to line.

\subsection{The initial estimate for $\mathcal{U}$}

Lemma 3.1. Let $g=g^{E}+h$ on $B_{\rho}$ be given. Then for each $\varepsilon_{0} \in[0,1)$ there exists $0<\rho_{0}<\rho$ and a constant $C$ with the following properties. If $\varepsilon<\varepsilon_{0}$ and $\Sigma$ is of Willmore type with multiplier $\lambda$ in $B_{r}$ with $r<\rho_{0}$ and 
1. $\Sigma$ is a topological sphere,

2. $\lambda \geq-\varepsilon /|\Sigma|$,

3. $H>0$ on $\Sigma$.

Then

$$
\begin{aligned}
& \int_{\Sigma}|\stackrel{\leftrightarrow}{A}|^{2}+|\nabla \log H|^{2} \mathrm{~d} \mu \leq C r^{2}+\varepsilon \\
& \left|\int_{\Sigma} H^{2} \mathrm{~d} \mu-16 \pi\right| \leq C\left(\varepsilon+r^{2}\right) \\
& |\Sigma| \leq C r^{2}, \quad \text { and } \\
& |\lambda| \leq C(1+\varepsilon /|\Sigma|) .
\end{aligned}
$$

Note that in equation (1.1) the term $\Delta H$ scales like $|\Sigma|^{-3 / 2}$ so that the assumption on $\lambda$ implies that the term $\lambda H$ is of the same order of magnitude as this leading order term but small in comparison.

The proof is similar to the proof of lemmas 3.1 and 3.3 in [6] although the role of the individual terms is somewhat different.

Proof. Multiplying equation (1.1) by $H^{-1}$ and integrating by parts gives

$$
\int_{\Sigma}|\nabla \log H|^{2}+|\stackrel{\AA}{A}|^{2}+\operatorname{Ric}(\nu, \nu)+\lambda \mathrm{d} \mu=0
$$

In view of the assumption on $\lambda$ and the fact that Ric is bounded this yields the estimate

$$
\int_{\Sigma}|\nabla \log H|^{2}+|\stackrel{\AA}{A}|^{2} \mathrm{~d} \mu \leq C|\Sigma|+\varepsilon
$$

Inserting this back into (3.1) we get

$$
|\lambda| \leq C\left(1+\varepsilon|\Sigma|^{-1}\right),
$$

which yields the last claim of the lemma.

Integrating (2.4) over $\Sigma$ and using (3.2) and the Gauss-Bonnet theorem we find that

$$
\left|\frac{1}{2} \int_{\Sigma} H^{2} \mathrm{~d} \mu-8 \pi\right| \leq C(|\Sigma|+\varepsilon) .
$$


In view of the area estimate from lemma 2.2, this yields an estimate of the form

$$
|\Sigma| \leq C r^{2} \int_{\Sigma} H^{2} \mathrm{~d} \mu \leq C r^{2}(1+|\Sigma|)
$$

If $r<\rho_{0}$ is chosen small enough, we can absorb the term $C r^{2}|\Sigma|$ on the right to the left and obtain the estimates

$$
\left|\int_{\Sigma} H^{2} \mathrm{~d} \mu-16 \pi\right| \leq C\left(\varepsilon+r^{2}\right)
$$

and

$$
|\Sigma| \leq C r^{2}
$$

which are the second and third claims. Plugging this into estimate (3.2), we obtain the remaining estimate.

\subsection{An improved estimate for $\mathcal{U}$}

The initial estimate from lemma 3.1 allows to apply the a priori estimates from section 3 in [6] to get higher order estimates and to improve on the initial estimate.

Theorem 3.2. Let $g=g^{E}+h$ on $B_{\rho}$ be given. Then there exist $\varepsilon>0$, $0<\rho_{0}<\rho$ and a constant $C$ with the following properties. If $\varepsilon<\varepsilon_{0}$ and $\Sigma$ is of Willmore type with multiplier $\lambda$ in $B_{r}$ with $r<\rho_{0}$ and

1. $\Sigma$ is a topological sphere,

2. $\lambda \geq-\varepsilon /|\Sigma|$,

3. $H>0$ on $\Sigma$.

Then

$$
\int_{\Sigma} \frac{\left|\nabla^{2} H\right|^{2}}{H^{2}}+|\nabla A|^{2}+|A|^{2}|\AA|^{2} \mathrm{~d} \mu \leq C \int_{\Sigma}|\omega|^{2}+(\operatorname{Ric}(\nu, \nu)+\lambda)^{2} \mathrm{~d} \mu .
$$

Here $\omega=\operatorname{Ric}(\nu, \cdot)^{T}$ is a the tangential/normal component of the ambient Ricci curvature.

Proof. This is a consequence of the calculation in section 3 of [6]. Note that the calculation there makes use of the fact that $\|\AA\|_{L^{2}}+\|\nabla \log H\|_{L^{2}}$ 
can be made arbitrarily small (cf. lemma 3.8 there) and this is where the initial estimate from section 3.1 enters here. In particular, theorem 3.9 in [6] implies that in the local situation we have

$$
\begin{aligned}
& \int_{\Sigma} \frac{\left|\nabla^{2} H\right|^{2}}{H^{2}}+|\nabla A|^{2}+|A|^{2}|\AA|^{2} \mathrm{~d} \mu \\
& \leq C \int_{\Sigma}|\omega|^{2}+(\operatorname{Ric}(\nu, \nu)+\lambda)^{2} \mathrm{~d} \mu \\
& \quad+C \sup _{B_{\rho}}|\operatorname{Ric}| \int_{\Sigma}|\AA|^{2}+|\nabla \log H|^{2} \mathrm{~d} \mu .
\end{aligned}
$$

Next we use the Michael-Simon-Sobolev inequality to estimate

$$
\int_{\Sigma}|\AA|^{2} \mathrm{~d} \mu \leq C\left(\int_{\Sigma}|\nabla \AA|+H|\AA| \mathrm{d} \mu\right)^{2} \leq C|\Sigma| \int_{\Sigma}|\nabla A|^{2}+H^{2}|\AA|^{2} \mathrm{~d} \mu .
$$

Similarly we get

$$
\begin{aligned}
\int_{\Sigma}|\nabla \log H|^{2} \mathrm{~d} \mu & \leq\left(\int_{\Sigma} \frac{\left|\nabla^{2} H\right|}{H}+|\nabla \log H|^{2}+|\nabla H| \mathrm{d} \mu\right)^{2} \\
& \leq C|\Sigma| \int_{\Sigma} \frac{|\nabla H|^{2}}{H^{2}}+|\nabla A|^{2}+|\nabla \log H|^{4} \mathrm{~d} \mu .
\end{aligned}
$$

Applying the Michael-Simon Sobolev inequality once more we have

$$
\begin{aligned}
& \int_{\Sigma}|\nabla \log H|^{4} \mathrm{~d} \mu \\
& \leq C\left(\int_{\Sigma} \frac{\left|\nabla^{2} H\right|}{H}|\nabla \log H|+|\nabla \log H|^{3}+H|\nabla \log H|^{2} \mathrm{~d} \mu\right)^{2} \\
& \leq C\|\nabla \log H\|_{L^{2}(\Sigma)}^{2} \int_{\Sigma} \frac{\left|\nabla^{2} H\right|^{2}}{H^{2}}+|\nabla \log H|^{4}+|\nabla A|^{2} \mathrm{~d} \mu .
\end{aligned}
$$

Using lemma 3.1 we know that for $\varepsilon$ and $r<r_{0}$ small enough we get

$$
\int_{\Sigma}|\nabla \log H|^{4} \mathrm{~d} \mu \leq C|| \nabla \log H \|_{L^{2}(\Sigma)}^{2} \int_{\Sigma} \frac{\left|\nabla^{2} H\right|^{2}}{H^{2}}+|\nabla A|^{2} \mathrm{~d} \mu .
$$

Inserting this into the above estimate for $\int_{\Sigma}|\nabla \log H|^{2} \mathrm{~d} \mu$ we conclude

$$
\int_{\Sigma}|\nabla \log H|^{2} \mathrm{~d} \mu \leq C|\Sigma| \int_{\Sigma} \frac{\left|\nabla^{2} H\right|^{2}}{H^{2}}+|\nabla A|^{2} \mathrm{~d} \mu .
$$

Hence we see that for $r<\rho_{0}$ small enough, we can absorb the second term on the right hand side of (3.3). 
The remaining task is to estimate the term on the right hand side in theorem 3.2. We start with the following calculation.

Lemma 3.3. Assume that the metric $g=g^{E}+h$ on $B_{\rho}$ is given. Then there exists a constant $C$ such that for all surfaces $\Sigma \subset B_{r}$ we have

$$
\left|\int_{\Sigma} \operatorname{Ric}(\nu, \nu) \mathrm{d} \mu-\frac{|\Sigma|}{3} \operatorname{Sc}(0)\right| \leq C|\Sigma|\left(\|\AA\|_{L^{2}(\Sigma)}+r\right)
$$

Proof. Note that if either $\|\AA\|_{L^{2}(\Sigma)}$ or $r$ is large, the estimate is trivially satisfied, so that it is sufficient to show it in the case where theorem 2.4 is applicable and we are furthermore allowed to assume that $0<r<1$. We use theorem 2.4 to approximate $\Sigma$ by a Euclidean sphere $S=S_{a^{E}}\left(R^{E}\right)$ with $a^{E} \in B_{r}(0)$ and $R^{E}=\sqrt{|\Sigma|^{E} / 4 \pi}$. Since

$$
\left.|| \Sigma\right|^{E}-|\Sigma|\left|\leq \int_{\Sigma}\right| \mathrm{d} \mu^{E}-\mathrm{d} \mu\left|\mathrm{d} \mu \leq C r^{2}\right| \Sigma \mid
$$

we infer that

$$
\left|R^{E}-R\right| \leq C r R
$$

where $R=\sqrt{|\Sigma| / 4 \pi}$. It is well known that

$$
\int_{S} \operatorname{Ric}_{0}(N, N) \mathrm{d} \mu^{E}=\frac{|\Sigma|^{E}}{3} \operatorname{Sc}(0)
$$

where we use the notation of theorem 2.4, that is $N$ is the Euclidean normal of $S$. Furthermore $\mathrm{Ric}_{0}$ denotes the Ricci-tensor of $M$ evaluated at the origin.

The first step is to estimate

$$
\begin{aligned}
& \left|\int_{S} \operatorname{Ric}(N, N) \mathrm{d} \mu^{E}-\int_{S} \operatorname{Ric}_{0}(N, N) \mathrm{d} \mu^{E}\right| \\
& \quad \leq|\Sigma|^{E} \sup _{p \in S}\left|\operatorname{Ric}_{p}(N, N)-\operatorname{Ric}_{0}(N, N)\right| \leq C|\Sigma|^{E} r
\end{aligned}
$$

and therefore

$$
\left|\int_{S} \operatorname{Ric}(N, N) \mathrm{d} \mu^{E}-\frac{|\Sigma|^{E}}{3} \operatorname{Sc}(0)\right| \leq C r|\Sigma| .
$$

In the next step we estimate $\int_{\Sigma} \operatorname{Ric}(\nu, \nu)$ in terms of $\int_{S} \operatorname{Ric}(N, N) \mathrm{d} \mu^{E}$. To this end note that

$$
\left|\int_{\Sigma} \operatorname{Ric}(\nu, \nu) \mathrm{d} \mu-\int_{\Sigma} \operatorname{Ric}(\nu, \nu) \mathrm{d} \mu^{E}\right| \leq C|\Sigma| r^{2} .
$$


The resulting integral can be evaluated using the conformal parametrization $\psi: S \rightarrow \Sigma$ from theorem 2.4. We can express

$$
\int_{\Sigma} \operatorname{Ric}(\nu, \nu) \mathrm{d} \mu^{E}=\int_{S} \operatorname{Ric} \circ \psi(\nu \circ \psi, \nu \circ \psi) h^{2} \mathrm{~d} \mu^{E}
$$

The estimates of theorem 2.4 and the Cauchy-Schwarz inequality imply that

$$
\begin{aligned}
& \left|\int_{\Sigma} \operatorname{Ric}(\nu, \nu) \mathrm{d} \mu^{E}-\int_{S} \operatorname{Ric}(N, N) \mathrm{d} \mu^{E}\right| \\
& \leq\left|\int_{S}(\operatorname{Ric} \circ \psi-\operatorname{Ric})(\nu \circ \psi, \nu \circ \psi) h^{2} \mathrm{~d} \mu^{E}\right| \\
& \quad+\left|\int_{S} \operatorname{Ric}(\nu \circ \psi-N, \nu \circ \psi) h^{2} \mathrm{~d} \mu^{E}\right| \\
& \quad+\left|\int_{S} \operatorname{Ric}(N, \nu \circ \psi-N) h^{2} \mathrm{~d} \mu^{E}\right|+\left|\int_{S} \operatorname{Ric}(N, N)\left(h^{2}-1\right) \mathrm{d} \mu^{E}\right| \\
& \leq C|\Sigma|\|\psi-\operatorname{id}\|_{L^{\infty}(S)}+C|\Sigma|^{1 / 2}\|\nu \circ \psi-N\|_{L^{2}(S)}+C|\Sigma|\left\|h^{2}-1\right\|_{L^{\infty}(S)} \\
& \leq C|\Sigma|\left\|\AA^{E}\right\|_{L^{2}\left(\Sigma, \gamma^{E}\right)} .
\end{aligned}
$$

In combination with lemma 2.5 we infer

$$
\left|\int_{\Sigma} \operatorname{Ric}(\nu, \nu) \mathrm{d} \mu^{E}-\int_{S} \operatorname{Ric}(N, N) \mathrm{d} \mu^{E}\right| \leq C|\Sigma|\left(\|\AA\|_{L^{2}(\Sigma)}+r^{2}\right) .
$$

Collecting all the above estimates results in the estimate

$$
\left|\int_{\Sigma} \operatorname{Ric}(\nu, \nu) \mathrm{d} \mu-\frac{|\Sigma|}{3} \operatorname{Sc}(0)\right| \leq C|\Sigma|\left(\|\AA\|_{L^{2}(\Sigma)}+r\right)
$$

which is precisely the claim.

In the following lemma we derive an estimate for the Lagrange parameter $\lambda$.

Lemma 3.4. Assume that the metric $g=g^{E}+h$ on $B_{\rho}$ is given. Then there exist $\varepsilon_{0}, r_{0}<\rho$ and a constant $C$ such that all surfaces $\Sigma \subset B_{r}$ as in the statement of theorem 3.2 with $\varepsilon<\varepsilon_{0}$ and $r<r_{0}$ satisfy

$$
\left|\lambda+\frac{1}{3} \operatorname{Sc}(0)\right| \leq C|\Sigma|^{-1}\left(\|\stackrel{\AA}{A}\|_{L^{2}(\Sigma)}^{2}+\|\nabla \log H\|_{L^{2}(\Sigma)}^{2}\right)+C r
$$

In particular

$$
|\lambda| \leq C|\Sigma|^{-1}\left(\|\AA\|_{L^{2}(\Sigma)}^{2}+\|\nabla \log H\|_{L^{2}(\Sigma)}^{2}\right)+C .
$$


Proof. Equation (3.1) implies that

$$
\left|\lambda+\frac{1}{|\Sigma|} \int_{\Sigma} \operatorname{Ric}(\nu, \nu) \mathrm{d} \mu\right| \leq|\Sigma|^{-1}\left(\|\AA\|_{L^{2}(\Sigma)}^{2}+\|\nabla \log H\|_{L^{2}(\Sigma)}^{2}\right) .
$$

Apply lemma 3.3 to calculate

$$
\left|\frac{1}{|\Sigma|} \int_{\Sigma} \operatorname{Ric}(\nu, \nu) \mathrm{d} \mu-\frac{1}{3} \operatorname{Sc}(0)\right| \leq C\left(\|\AA\|_{L^{2}(\Sigma)}+r\right)
$$

and note that

$$
\|\AA\|_{L^{2}(\Sigma)}=|\Sigma|^{1 / 2}|\Sigma|^{-1 / 2}\|\AA\|_{L^{2}(\Sigma)} \leq \frac{1}{2}|\Sigma|+\frac{1}{2}|\Sigma|^{-1}\|\AA\|_{L^{2}(\Sigma)}^{2} .
$$

Since $|\Sigma| \leq C r^{2}$ by lemma 3.1, this implies the claim in combination with equation (3.7).

Theorem 3.5. Assume that the metric $g=g^{E}+h$ on $B_{\rho}$ is given. Then there exist $\varepsilon_{0}>0, r_{0}<\rho$ and a constant $C$ such that all surfaces $\Sigma \subset B_{r}$ as in the statement of theorem 3.2 with $\varepsilon<\varepsilon_{0}$ and $r<r_{0}$ satisfy

$$
\int_{\Sigma} \frac{\left|\nabla^{2} H\right|^{2}}{H^{2}}+|\nabla A|^{2}+|A|^{2}|\AA|^{2} \mathrm{~d} \mu \leq C|\Sigma| .
$$

Proof. In view of theorem 3.2 and the fact that Ric and $\omega$ are bounded, we infer the estimate

$$
\int_{\Sigma} \frac{\left|\nabla^{2} H\right|^{2}}{H^{2}}+|\nabla A|^{2}+|A|^{2}|\AA|^{2} \mathrm{~d} \mu \leq C|\Sigma|\left(1+\lambda^{2}\right) .
$$

The crucial term to estimate thus is $\lambda^{2}|\Sigma|$. We use the estimate from lemma 3.4 to get

$$
\lambda^{2}|\Sigma| \leq C|\Sigma|^{-1}\left(\|\AA\|_{L^{2}(\Sigma)}^{4}+\|\nabla \log H\|_{L^{2}(\Sigma)}^{4}\right)+C|\Sigma| .
$$

Combining (3.4) and (3.6) with (3.8) and (3.9), we infer

$$
\begin{aligned}
& \int_{\Sigma} \frac{\left|\nabla^{2} H\right|^{2}}{H^{2}}+|\nabla A|^{2}+|A|^{2}|\AA|^{2} \mathrm{~d} \mu \\
& \quad \leq C|\Sigma|+C|\Sigma|\left(\int_{\Sigma} \frac{\left|\nabla^{2} H\right|^{2}}{H^{2}}+|\nabla A|^{2}+|A|^{2}|\AA|^{2} \mathrm{~d} \mu\right)^{2} .
\end{aligned}
$$

To proceed note that by equation (3.8) and lemma 3.1 we find that

$$
\int_{\Sigma} \frac{\left|\nabla^{2} H\right|^{2}}{H^{2}}+|\nabla A|^{2}+|A|^{2}|\AA|^{2} \mathrm{~d} \mu \leq C|\Sigma|\left(1+\lambda^{2}\right) \leq C\left(|\Sigma|+\varepsilon^{2}|\Sigma|^{-1}\right)
$$


Using this in equation (3.10) to estimate part of the right hand side, we get

$$
\begin{aligned}
& \int_{\Sigma} \frac{\left|\nabla^{2} H\right|^{2}}{H^{2}}+|\nabla A|^{2}+|A|^{2}|\AA|^{2} \mathrm{~d} \mu \\
& \quad \leq C|\Sigma|+C\left(|\Sigma|^{2}+\varepsilon^{2}\right)\left(\int_{\Sigma} \frac{\left|\nabla^{2} H\right|^{2}}{H^{2}}+|\nabla A|^{2}+|A|^{2}|\AA|^{2} \mathrm{~d} \mu\right) .
\end{aligned}
$$

Thus choosing $0<\varepsilon$ and $r<r_{0}$ small enough, we can absorb the second term on the right to the left and infer the claimed estimate.

Corollary 3.6. Assume that the metric $g=g^{E}+h$ on $B_{\rho}$ is given. Then there exist $\varepsilon_{0}>0,0<r_{0}<\rho$ and a constant $C$ and such that all surfaces $\Sigma \subset B_{r}$ as in the statement of theorem 3.2 with $\varepsilon<\varepsilon_{0}$ and $r<r_{0}$ satisfy

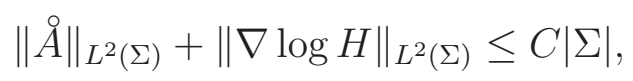

and

$$
\left|\lambda+\frac{1}{3} \operatorname{Sc}(0)\right| \leq C r
$$

Proof. The first claim follows from (3.4), (3.6) and theorem 3.5, whereas the second claim follows from the first one in view of lemma 3.4 .

Note that this corollary yields the first claim in theorem 1.1.

\subsection{Estimates in the $L^{\infty}$-norm}

To proceed further we need an estimate for the size of $H^{-1}$ in the $L^{\infty}$-norm. To this end we recall lemma 4.7 from [6].

Lemma 3.7. Assume that the metric $g=g^{E}+h$ on $B_{\rho}$ is given. Then there exist $\varepsilon_{0}>0, r_{0}<\rho$ and a constant $C<\infty$ such that for all surfaces $\Sigma \subset B_{r}$ as in the statement of theorem 3.2 with $\varepsilon<\varepsilon_{0}$ and $r<r_{0}$ and for all smooth forms $\phi$ on $\Sigma$ we have

$$
\|\phi\|_{L^{\infty}(\Sigma)}^{4} \leq C\|\phi\|_{L^{2}(\Sigma)}^{2} \int_{\Sigma}\left|\nabla^{2} \phi\right|^{2}+|H|^{4}|\phi|^{2} \mathrm{~d} \mu .
$$

Proof. This lemma is a variant of [5, Lemma 2.8]. The proof from there can be carried over to our situation, since it mainly relies on the Michael-SimonSobolev inequality which is also available in this situation, cf. lemma 2.3. 
Proposition 3.8. Assume that the metric $g=g^{E}+h$ on $B_{\rho}$ is given. Then there exist $\varepsilon_{0}>0, r_{0}<\rho$ and a constant $C<\infty$ such that for all surfaces $\Sigma \subset B_{r}$ as in the statement of theorem 3.2 with $\varepsilon<\varepsilon_{0}$ and $r<r_{0}$ we have

$$
\left\|H^{-1}\right\|_{L^{\infty}(\Sigma)} \leq C|\Sigma|^{1 / 2}
$$

Proof. The idea is to apply lemma 3.7 to the function $H^{-1}$. We thus estimate

$$
\left\|H^{-1}\right\|_{L^{2}(\Sigma)}^{2} \leq|\Sigma|\left\|H^{-1}\right\|_{L^{\infty}(\Sigma)}^{2}
$$

and calculate

$$
\nabla^{2}\left(H^{-1}\right)=-H^{-2} \nabla^{2} H+2 H^{-3} \nabla H \otimes \nabla H .
$$

Thus

$$
\begin{aligned}
\int_{\Sigma}\left|\nabla^{2}\left(H^{-1}\right)\right|^{2} \mathrm{~d} \mu & \leq C \int_{\Sigma} H^{-6}|\nabla H|^{4}+H^{-4}\left|\nabla^{2} H\right|^{2} \mathrm{~d} \mu \\
& \leq C\left\|H^{-1}\right\|_{L^{\infty}(\Sigma)}^{2} \int_{\Sigma} \frac{\left|\nabla^{2} H\right|^{2}}{H^{2}}+|\nabla \log H|^{4} \mathrm{~d} \mu .
\end{aligned}
$$

From (3.5) we get for $\varepsilon$ and $r$ small enough

$$
\int_{\Sigma}|\nabla \log H|^{4} \mathrm{~d} \mu \leq C\left(\int_{\Sigma}|\nabla \log H|^{2} \mathrm{~d} \mu\right)\left(\int_{\Sigma} \frac{\left|\nabla^{2} H\right|^{2}}{H^{2}}+|\nabla A|^{2} \mathrm{~d} \mu\right)
$$

By theorem 3.5 and corollary 3.6 we therefore conclude

$$
\int_{\Sigma}|\nabla \log H|^{4} \mathrm{~d} \mu \leq C|\Sigma|^{3} .
$$

Together with equation (3.12) and theorem 3.5 this yields

$$
\int_{\Sigma}\left|\nabla^{2}\left(H^{-1}\right)\right|^{2} \mathrm{~d} \mu \leq C\left\|H^{-1}\right\|_{L^{\infty}(\Sigma)}^{2}|\Sigma| .
$$

Plugging estimates (3.14) and (3.11) into the estimate from lemma 3.7, we find that

$$
\begin{aligned}
\left\|H^{-1}\right\|_{L^{\infty}(\Sigma)}^{4} & \leq C|\Sigma|\left\|H^{-1}\right\|_{L^{\infty}(\Sigma)}^{2}\left(\left\|H^{-1}\right\|_{L^{\infty}(\Sigma)}^{2}|\Sigma|+\int_{\Sigma} H^{2} \mathrm{~d} \mu\right) \\
& \leq C|\Sigma|^{2}\left\|H^{-1}\right\|_{L^{\infty}(\Sigma)}^{4}+C|\Sigma|\left\|H^{-1}\right\|_{L^{\infty}(\Sigma)}^{2} \\
& \leq\left(C|\Sigma|^{2}+\frac{1}{2}\right)\left\|H^{-1}\right\|_{L^{\infty}(\Sigma)}^{4}+C|\Sigma|^{2} .
\end{aligned}
$$

If $r<r_{0}$ and thus $|\Sigma|$ is small, the first term on the right can be absorbed, and the claim follows. 


\section{Proof of Theorem 1.1}

This section is devoted to the proof of theorem 1.1. Note that the first claim already follows from corollary 3.6, hence it remains to show the second claim.

Throughout this section we assume that the surface $\Sigma$ in question is of Willmore type with multiplier $\lambda$. We assume further that $H>0$ on $\Sigma$, $\lambda \geq-\varepsilon|\Sigma|^{-1}$ and $\Sigma \subset B_{r}(0)$ where $\varepsilon<\varepsilon_{0}$ and $r<r_{0}$. Here $\varepsilon_{0}>0$ and $r_{0}>0$ are chosen so that all the estimates from section 3 are applicable.

To get started we recall the splitting (2.5) of the Willmore functional:

$$
\mathcal{W}(\Sigma)=8 \pi(1-q(\Sigma))+\mathcal{U}(\Sigma)+\mathcal{V}(\Sigma)
$$

Since the first term on the right is a topological constant we infer that the variation of $\mathcal{W}$, when $\Sigma$ is varied by the normal vector field $f \nu$ for $f \in C^{\infty}(\Sigma)$, satisfies

$$
\delta_{f} \mathcal{W}(\Sigma)=\delta_{f} \mathcal{U}(\Sigma)+\delta_{f} \mathcal{V}(\Sigma)
$$

Equation (1.1) implies that the variation of $\mathcal{W}$ is given by

$$
\delta_{f} \mathcal{W}(\Sigma)=\lambda \int_{\Sigma} H f \mathrm{~d} \mu,
$$

whenever $\Sigma$ is of Willmore type with multiplier $\lambda$. Thus on such a surface we have

$$
\lambda \int_{\Sigma} H f \mathrm{~d} \mu=\delta_{f} \mathcal{U}(\Sigma)+\delta_{f} \mathcal{V}(\Sigma)
$$

We shall evaluate these terms when the normal velocity $f$ of the variation is given by

$$
f=H^{-1} g(b, \nu)
$$

where $b \in \mathbf{R}^{3}$ is a fixed vector with $|b|=1$. We start with the left hand side of equation (4.2).

\subsection{The left hand side of (4.2)}

We have

$$
\int_{\Sigma} H f \mathrm{~d} \mu=\int_{\Sigma} g(b, \nu) \mathrm{d} \mu .
$$


To evaluate this expression note that since $\Sigma$ is assumed to be a topological sphere in $B_{\rho}$ it must bound a region $\Omega$. We wish to estimate the volume of $\Omega$. To this end, we approximate $\Sigma$ by a Euclidean sphere $S=S_{R^{E}}\left(a^{E}\right)$. With $x$ the position vector field in $B_{\rho}$ we define the vector field

$$
X=x-a^{E}
$$

such that

$$
\operatorname{div}_{g^{E}} X=3,
$$

in $\Omega$. On $\Sigma$ we have

$$
\begin{aligned}
|X| & =\left|\psi-a^{E}\right| \leq\left\|\psi-\mathrm{id}_{S}\right\|_{L^{\infty}(S)}+\left\|\mathrm{id}_{S}-a^{E}\right\|_{L^{\infty}(S)} \\
& \leq C|\Sigma|^{1 / 2}\left(1+\left\|\AA^{E}\right\|_{L^{2}\left(\Sigma, g^{E}\right)}\right)
\end{aligned}
$$

since $\left\|\operatorname{id}_{S}-a^{E}\right\|_{L^{\infty}(S)}=R^{E}$. Here $\operatorname{id}_{S}: S \rightarrow B_{\rho}$ denotes the standard embedding of $S$ into $B_{\rho}$.

We integrate the relation (4.5) over $\Omega$ and use partial integration to conclude that

$$
3 \mathrm{Vol}^{E}(\Omega)=\int_{\Sigma} g^{E}\left(X, \nu^{E}\right) \mathrm{d} \mu^{E} .
$$

Replacing the integral over $\Sigma$ by an integral over $S$ introduces an error of the form

$$
\begin{aligned}
& \left|\int_{\Sigma} g^{E}\left(X, \nu^{E}\right) \mathrm{d} \mu^{E}-\int_{S} g^{E}(X, N) \mathrm{d} \mu^{E}\right| \\
& \quad \leq C\left(|\Sigma|\|\psi-\mathrm{id}\|_{L^{\infty}(S)}+|\Sigma|^{3 / 2}\left\|h^{2}-1\right\|_{L^{\infty}(S)}+|\Sigma|\left\|\nu^{E} \circ \psi-N\right\|_{L^{2}(S)}\right) \\
& \quad \leq C|\Sigma|^{3 / 2}\left\|\AA^{E}\right\|_{L^{2}\left(\Sigma, g^{E}\right)} .
\end{aligned}
$$

In view of lemma 2.5 we thus obtain the estimate

$$
\left|\operatorname{Vol}^{E}(\Omega)-\frac{|\Sigma|^{3 / 2}}{6 \pi^{1 / 2}}\right| \leq C|\Sigma|^{3 / 2}\left(\|\AA\|_{L^{2}(\Sigma)}+r^{2}\right) .
$$

The assumption (2.2) implies that for the volume elements of $g$ and $g^{E}$ we have that

$$
\left|\mathrm{d} V_{g}-\mathrm{d} V_{g^{E}}\right| \leq C|x|^{2} .
$$

Combining the last two estimates we get

$$
\left|\operatorname{Vol}(\Omega)-\frac{|\Sigma|^{3 / 2}}{6 \pi^{1 / 2}}\right| \leq C|\Sigma|^{3 / 2}\left(\|\AA\|_{L^{2}(\Sigma)}+r^{2}\right) .
$$


Using corollary 3.6 we finally conclude

$$
\left|\operatorname{Vol}(\Omega)-\frac{|\Sigma|^{3 / 2}}{6 \pi^{1 / 2}}\right| \leq C r^{2}|\Sigma|^{3 / 2}
$$

The right hand side of (4.4) can be expressed as a volume integral

$$
\int_{\Sigma} g(b, \nu) \mathrm{d} \mu=\int_{\Omega} \operatorname{div}_{M} b \mathrm{~d} V
$$

and since $|\nabla b| \leq C r$ we estimate

$$
\left|\int_{\Omega} \operatorname{div}_{M} b \mathrm{~d} V\right| \leq C r \operatorname{Vol}(\Omega) \leq C r|\Sigma|^{3 / 2}
$$

Thus, since $\lambda$ is bounded in view of corollary 3.6, we obtain

$$
\left|\lambda \int_{\Sigma} H f \mathrm{~d} \mu\right| \leq C r|\Sigma|^{3 / 2}
$$

\subsection{The variation of $\mathcal{U}$}

A fairly straight forward calculation shows that the variation of $\mathcal{U}$ is given by

$$
\delta_{f} \mathcal{U}(\Sigma)=-\int_{\Sigma} 2\left\langle\AA, \nabla^{2} f\right\rangle+2 f\left\langle\AA, \operatorname{Ric}^{T}\right\rangle+f H|\AA|^{2} \mathrm{~d} \mu,
$$

where $\operatorname{Ric}^{T}$ denotes the tangential projection of the Ricci curvature of $M$ onto $\Sigma$. With $f$ as in equation (4.3), the second and third term are easily bounded as follows

$$
\begin{aligned}
& \int_{\Sigma} 2 f\left\langle\AA, \operatorname{Ric}^{T}\right\rangle+f H|\AA|^{2} \mathrm{~d} \mu \\
& \quad \leq C|\Sigma|^{1 / 2} \sup _{\Sigma}|f|\left(\int_{\Sigma}|\AA|^{2} \mathrm{~d} \mu\right)^{1 / 2}+C \int_{\Sigma}|\AA|^{2} \mathrm{~d} \mu \leq C|\Sigma|^{2}
\end{aligned}
$$

where we used the fact that $|g(b, \nu)| \leq C$ together with corollary 3.6 and proposition 3.8.

To treat the first term in (4.9), we calculate the first and second derivatives of $f$. Choosing a local ON-frame $\left\{e_{1}, e_{2}\right\}$ on $\Sigma$ we obtain

$$
\nabla_{e_{i}} f=H^{-1} g\left(\nabla_{e_{i}} b, \nu\right)+H^{-1} g\left(b, e_{j}\right) A_{i}^{j}-H^{-2} \nabla_{e_{i}} H g(b, \nu)
$$


and thus in view of the estimates from theorem 3.5, proposition 3.8 and the fact that $|\nabla b| \leq C r$, we find

$$
\int_{\Sigma}|\nabla f|^{2} \mathrm{~d} \mu \leq C r^{2}
$$

Differentiating equation (4.11) once more, we obtain

$$
\begin{aligned}
\nabla_{e_{i}} & \nabla_{e_{j}} f \\
= & -A_{i}^{k} A_{j k} f+2 H^{-3} \nabla_{e_{i}} H \nabla_{e_{j}} H g(b, \nu)-H^{-2} \nabla_{i, j}^{2} H g(b, \nu) \\
& +H^{-1}\left(g\left(\nabla_{e_{i}} \nabla_{e_{j}} b, \nu\right)+g\left(\nabla_{e_{i}} b, e_{k}\right) A_{j}^{k}+g\left(\nabla_{e_{j}} b, e_{k}\right) A_{i}^{k}+\nabla_{e_{j}} A_{i}^{k} g\left(b, e_{k}\right)\right) \\
& -H^{-2}\left(\nabla_{e_{i}} H\left(g\left(\nabla_{e_{j}} b, \nu\right)+g\left(b, e_{k}\right) A_{j}^{k}\right)+\nabla_{e_{j}} H\left(g\left(\nabla_{e_{i}} b, \nu\right)+g\left(b, e_{k}\right) A_{i}^{k}\right)\right) .
\end{aligned}
$$

Our goal is to estimate $\int_{\Sigma}\left\langle\AA, \nabla^{2} f\right\rangle \mathrm{d} \mu$ so that all we need of $\nabla^{2} f$ is its traceless part. Note that the largest term in expression (4.12) is the first one on the right hand side. Its contribution consists mainly of the trace part. When removing the trace, we find that we can estimate

$$
\begin{aligned}
\left|\left(\nabla^{2} f\right)^{\circ}\right| \leq C & \left(|A||\AA||f|+H^{-1}\left|\nabla^{2} b\right|+H^{-1}|A||\nabla b|+H^{-1}|\nabla A|\right. \\
& \left.+H^{-2}|\nabla H||\nabla b|+H^{-2}|\nabla H||A|+H^{-2}\left|\nabla^{2} H\right|+H^{-3}|\nabla H|^{2}\right) .
\end{aligned}
$$

In view of the fact that $|\nabla b| \leq C r$ and $\left|\nabla^{2} b\right| \leq C$, and using the estimates from theorem 3.5, corollary 3.6 and proposition 3.8, we infer that

$$
\int_{\Sigma}\left|\left(\nabla^{2} f\right)^{\circ}\right|^{2} \mathrm{~d} \mu \leq C r^{2}|\Sigma|
$$

Here we also used that by the estimates from section 3, (3.13) and the fact that $|A|^{2}=\frac{1}{2} H^{2}+|\stackrel{\AA}{A}|^{2}$ we have

$$
\begin{aligned}
\int_{\Sigma} H^{-4}|\nabla H|^{2}|A|^{2} \mathrm{~d} \mu & \leq C\left(\int_{\Sigma}|\nabla \log H|^{4} \mathrm{~d} \mu\right)^{1 / 2}\left(\int_{\Sigma} H^{-4}\left(H^{4}+|\AA|^{4}\right) \mathrm{d} \mu\right)^{1 / 2} \\
& \leq C|\Sigma|^{3 / 2}\left(|\Sigma|^{1 / 2}+|\Sigma|^{3 / 2}\right) \leq C|\Sigma|^{2} .
\end{aligned}
$$

In view of the Cauchy-Schwarz inequality, corollary 3.6 and estimate (4.13) we infer that

$$
\int_{\Sigma}\left\langle\AA, \nabla^{2} f\right\rangle \mathrm{d} \mu \leq\left(\int_{\Sigma}|\AA|^{2} \mathrm{~d} \mu\right)^{1 / 2}\left(\int_{\Sigma}\left|\left(\nabla^{2} f\right)^{\circ}\right|^{2} \mathrm{~d} \mu\right)^{1 / 2} \leq C r|\Sigma|^{3 / 2} .
$$

Collecting the estimates (4.10) and (4.14) implies the desired bound on (4.9), namely

$$
\left|\delta_{f} \mathcal{U}(\Sigma)\right| \leq C r|\Sigma|^{3 / 2} .
$$




\subsection{The variation of $\mathcal{V}(\Sigma)$}

In section 5.5 of [6] the following expression for $\delta_{f} \mathcal{V}(\Sigma)$ was derived:

$$
\delta_{f} \mathcal{V}(\Sigma)=\int_{\Sigma}-f H G(\nu, \nu)-\frac{1}{2} f H \mathrm{Sc}+2 f\left\langle\AA, G^{T}\right\rangle-2 \omega(\nabla f) \mathrm{d} \mu
$$

where as before $G=\operatorname{Ric}-\frac{1}{2} g$ denotes the Einstein tensor of $M$ and $\omega=$ $\operatorname{Ric}(\nu, \cdot)^{T}$. Recall that we chose $f=H^{-1} g(b, \nu)$ above. In the expression (4.11) we split $A=\AA+\frac{1}{2} H \gamma$ and obtain

$$
\nabla_{e_{i}} f=\frac{1}{2} g\left(b, e_{i}\right)+H^{-1} g\left(\nabla_{e_{i}} b, \nu\right)+H^{-1} \stackrel{\leftrightarrow}{i}_{i}^{j} g\left(b, e_{j}\right)-H^{-2} \nabla H g(b, \nu) .
$$

Plugging this expression into equation (4.16) yields

$$
\begin{aligned}
\delta_{f} \mathcal{V}(\Sigma)= & \int_{\Sigma}-G(b, \nu)-\frac{1}{2} g(b, \nu) \mathrm{Sc}+2 f\left\langle\AA \AA^{\top}, G^{T}\right\rangle \\
& -2 w\left(e_{i}\right)\left(H^{-1} g\left(\nabla_{e_{i}} b, \nu\right)+H^{-1} \AA_{i}^{j} g\left(b, e_{j}\right)-H^{-2} \nabla H g(b, \nu)\right) \mathrm{d} \mu .
\end{aligned}
$$

Using theorem 3.5, corollary 3.6 and proposition 3.8 we estimate

$$
\begin{aligned}
& \mid \int_{\Sigma} 2 f\left\langle\AA, G^{T}\right\rangle-2 w\left(e_{i}\right)\left(H^{-1} g\left(\nabla_{e_{i}} b, \nu\right)\right. \\
& \left.\quad+H^{-1} \stackrel{\AA}{A}_{i}^{j} g\left(b, e_{j}\right)-H^{-2} \nabla H g(b, \nu)\right)\left.\mathrm{d} \mu|\leq C r| \Sigma\right|^{3 / 2} .
\end{aligned}
$$

In [6] the Pohozaev-Identity was used to estimate the term $\int_{\Sigma} G(b, \nu) \mathrm{d} \mu$. This is not really necessary here, the following simpler approach is sufficient. Recall that the divergence of $G$ with respect to the $g$-metric is zero due to the Bianchi-identity. Define the vector field $X$ by the requirement that $g(X, Y)=G(b, Y)$ for all vector fields $Y$ on $B_{\rho}$. Then the fact that $G$ is divergence free implies that

$$
\operatorname{div}_{M} X=\langle G, \nabla b\rangle .
$$

In section 4.1 we used that $\Sigma$ bounds a region $\Omega$ with $\operatorname{Vol}(\Omega) \leq C|\Sigma|^{3 / 2}$. To proceed, we integrate the relation (4.19) over $\Omega$ and after integration by parts we get (recall that $|\nabla b| \leq C r$ )

$$
\left|\int_{\Sigma} G(b, \nu) \mathrm{d} \mu\right|=\left|\int_{\Omega} \operatorname{div}_{M} X \mathrm{~d} V\right| \leq C r \operatorname{Vol}(\Omega) \leq C r|\Sigma|^{3 / 2} .
$$


In combination with equation (4.17) and estimate (4.18) we infer that

$$
\left|\delta_{f} \mathcal{V}(\Sigma)+\frac{1}{2} \int_{\Sigma} g(b, \nu) \operatorname{Scd} \mu\right| \leq C r|\Sigma|^{3 / 2}
$$

The final task is to estimate $\int_{\Sigma} g(b, \nu) \mathrm{Sc} \mathrm{d} \mu$. As before, we express this surface integral as a volume integral. To this end, we consider the vector field $X=$ Sc $b$ and calculate

$$
\operatorname{div}_{M} X=g(b, \nabla \mathrm{Sc})+\operatorname{Sc}_{\operatorname{div}} b .
$$

Since $\nabla \mathrm{Sc}=\nabla \mathrm{Sc}(0)+O(r), \nabla b=O(r)$ and $g=g^{E}+O\left(r^{2}\right)$ we infer

$$
\operatorname{div}_{M} X=g^{E}(b, \nabla \operatorname{Sc}(0))+O(r) .
$$

Thus we can calculate

$$
\int_{\Sigma} g(b, \nu) \operatorname{Scd} \mu=\int_{\Omega} \operatorname{div}_{M} X \mathrm{~d} V=\operatorname{Vol}(\Omega) g^{E}(b, \nabla \operatorname{Sc}(0))+O(r \operatorname{Vol}(\Omega)) .
$$

This finally leaves us with the estimate

$$
\left|\delta_{f} \mathcal{V}(\Sigma)+\frac{1}{2} \operatorname{Vol}(\Omega) g^{E}(b, \nabla \operatorname{Sc}(0))\right| \leq C r|\Sigma|^{3 / 2}
$$

\subsection{The conclusion}

To prove theorem 1.1, we combine the results from the previous sections. Combining equation (4.2) with the estimates (4.8) and (4.15) yields that for $f$ as in (4.3) we have

$$
\left|\delta_{f} \mathcal{V}(\Sigma)\right| \leq C r|\Sigma|^{3 / 2}
$$

In combination with (4.20) this gives

$$
\left|\operatorname{Vol}(\Omega) g^{E}(b, \nabla \operatorname{Sc}(0))\right| \leq C r|\Sigma|^{3 / 2},
$$

and since $\operatorname{Vol}(\Omega) \geq C^{-1}|\Sigma|^{3 / 2}$ we infer

$$
\left|g^{E}(b, \nabla \mathrm{Sc}(0))\right| \leq C r .
$$

Setting $b=\nabla \mathrm{Sc}(0) /|\nabla \mathrm{Sc}(0)|^{E}$ finally shows that

$$
|\nabla \operatorname{Sc}(0)|^{E} \leq C r
$$

Since we can let $r \rightarrow 0$ by the assumptions of theorem 1.1, we infer the claim, namely that $\nabla \mathrm{Sc}(0)=0$. 


\section{Expansion of the Willmore functional}

In this section we calculate the expansion of the Willmore functional on small surfaces using the estimates from section 3. We wish to emphasize here that similar expansions for the Willmore functional have been computed previously for geodesic spheres in [4, Section 3], where also the subsequent term in the expansion is calculated, and for perturbations of geodesic spheres in $[8]$.

The calculation here has the advantage that it works under much more general conditions. Namely we have the following theorem which holds in particular for surfaces as in theorem 1.1 due to the estimates of lemma 3.1 and corollary 3.6.

Theorem 5.1. Let $g=g^{E}+h$ on $B_{\rho}$ be given and let $c<\infty$ be a constant. Then there exists a constant $C$ depending only on $c, \rho$ and $h_{0}$ as in equation (2.2), such that the following holds.

Let $\Sigma \subset B_{r}$ be a spherical surface with $r<\rho$ such that

$$
\mathcal{U}(\Sigma) \leq c r|\Sigma| \quad \text { and } \quad|\Sigma| \leq c r .
$$

Then the following estimate holds:

$$
\left|\mathcal{W}(\Sigma)-8 \pi+\frac{|\Sigma|}{3} \operatorname{Sc}(0)\right| \leq C r|\Sigma| .
$$

Proof. We use the Gauss equation to express the Willmore functional as in equation $(2.5)$

$$
\mathcal{W}(\Sigma)=8 \pi+\mathcal{U}(\Sigma)+\mathcal{V}(\Sigma)
$$

By the first assumption the term $\mathcal{U}(\Sigma)$ is a lower order term and can be neglected. Furthermore

$$
\mathcal{V}(\Sigma)=2 \int_{\Sigma} G(\nu, \nu) \mathrm{d} \mu=\int_{\Sigma} 2 \operatorname{Ric}(\nu, \nu)-\operatorname{Sc} \mathrm{d} \mu .
$$

In view of the assumptions of the theorem, lemma 3.3 implies that

$$
\left|\int_{\Sigma} \operatorname{Ric}(\nu, \nu) \mathrm{d} \mu-\frac{|\Sigma|}{3} \operatorname{Sc}(0)\right| \leq C r|\Sigma| .
$$

Since $\mathrm{Sc}=\mathrm{Sc}(0)+O(r)$ we furthermore have

$$
\int_{\Sigma} \operatorname{Scd} \mu=|\Sigma| \operatorname{Sc}(0)+O(r|\Sigma|)
$$


so that in combination

$$
\mathcal{V}(\Sigma)=-\frac{|\Sigma|}{3} \mathrm{Sc}(0)+O(r|\Sigma|)
$$

Altogether this yields

$$
\mathcal{W}(\Sigma)=8 \pi-\frac{|\Sigma|}{3} \operatorname{Sc}(0)+O(r|\Sigma|),
$$

which is the desired expansion.

Corollary 5.2. Let $g$ be as in theorem 5.1 and assume that $\Sigma$ is a spherical surface in $B_{r}$ satisfying

$$
\mathcal{U}(\Sigma) \leq c|\Sigma|^{2} \quad \text { and } \quad|\Sigma| \leq c r .
$$

If $\Omega$ denotes the region bounded by $\Sigma$, then the Hawking mass of $\Sigma$,

$$
m_{H}(\Sigma)=\frac{|\Sigma|^{1 / 2}}{(16 \pi)^{3 / 2}}(16 \pi-2 \mathcal{W}(\Sigma))
$$

satisfies

$$
\frac{m_{H}(\Sigma)}{\operatorname{Vol}(\Omega)}=\frac{\operatorname{Sc}(0)}{16 \pi}+O(r) .
$$

Proof. This is a simple consequence of the expansion in theorem 5.1 which holds also under the stronger assumption of the corollary. In addition we use the fact that the volume of $\Omega$ satisfies

$$
\left|\operatorname{Vol}(\Omega)-\frac{|\Sigma|^{3 / 2}}{6 \pi^{1 / 2}}\right| \leq C r|\Sigma|^{3 / 2}
$$

which follows from equation (4.6).

\section{References}

[1] C. De Lellis and S. Müller. Optimal rigidity estimates for nearly umbilical surfaces. J. Differential Geom., 69(1):75-110, 2005.

[2] C. De Lellis and S. Müller. A $C^{0}$ estimate for nearly umbilical surfaces. Calc. Var. Partial Differential Equations, 26(3):283-296, 2006.

[3] O. Druet. Sharp local isoperimetric inequalities involving the scalar curvature. Proc. Amer. Math. Soc., 130(8):2351-2361 (electronic), 2002. 
[4] X.-Q. Fan, Y. Shi, and L.-F. Tam. Large-sphere and small-sphere limits of the Brown-York mass. Comm. Anal. Geom., 17(1):37-72, 2009.

[5] E. Kuwert and R. Schätzle. The Willmore flow with small initial energy. J. Differential Geom., 57(3):409-441, 2001.

[6] T. Lamm, J. Metzger, and F. Schulze. Foliations of asymptotically flat manifolds by surfaces of Willmore type, 2009.

[7] J. H. Michael and L. M. Simon. Sobolev and mean-value inequalities on generalized submanifolds of $\mathbf{R}^{n}$. Comm. Pure Appl. Math., 26:361-379, 1973.

[8] A. Mondino. Some results about the existence of critical points for the Willmore functional. to appear in Math. Z., 2009.

[9] S. Nardulli. The isoperimetric profile of a smooth Riemannian manifold for small volumes. Ann. Global Anal. Geom., 36(2):111-131, 2009.

[10] R. Schoen and S.-T. Yau. Lectures on Differential Geometry. Conference Proceedings and Lecture Notes in Geometry and Topology. International Press, Boston, 1994.

[11] L. Simon. Existence of surfaces minimizing the Willmore functional. Comm. Anal. Geom., 1(2):281-326, 1993.

[12] J. L. Weiner. On a problem of Chen, Willmore, et al. Indiana Univ. Math. J., 27(1):19-35, 1978.

[13] R. Ye. Foliation by constant mean curvature spheres. Pacific J. Math., 147(2):381-396, 1991.

[14] R. Ye. Constant mean curvature foliation: singularity structure and curvature estimate. Pacific J. Math., 174(2):569-587, 1996. 